\title{
Expression of YAP in the Skin Lesions of Lichen Planus
}

\author{
Jinjing Jia, Yan Zheng*, Jing Zhang, Zhengxiao Li, Jun Zhou, Zhaoxia Ying \\ Department of Dermatology, The Second Affiliated Hospital, School of Medicine, Xi'an Jiaotong University, China
}

Received: January 04, 2016; Accepted: January 11, 2016; Published: January 23, 2016

*Corresponding author: Yan Zheng, Department of Dermatology, The Second Affiliated Hospital, School of Medicine, Xi'an Jiaotong University, 157 Xiwu Road, Xi'an 710004, China. E-mail: zenyan66@126.com

\begin{abstract}
Objective: To explore the expressions of yes-associated protein (YAP) in skin lesions of lichen planus (LP) and normal skin.

Methods: Immunohistochemistry was carried out to quantify the expression of YAP in tissues from the lesions of 30 patients with LP and 30 healthy controls.

Results: In normal skin tissues, YAP was slightly expressed in the basal layers of the epidermis, and the positive rate was $26.67 \%$ (8/30). In LP, YAP was strongly expressed in the lower layers of the stratum spinosum and some of the infiltrating lymphocytes, and the positive rate was $56.67 \%(17 / 30)$. The expression of YAP in LP was significantly higher than that of normal skin $(P<0.05)$.
\end{abstract}

Conclusions: YAP may be involved in the occurrence and development of LP.

Keywords: Yes-associated protein; Lichen Planus; Hippo signaling pathway; Oncogene

\section{Introduction}

Lichen Planus (LP) is chronic inflammatory disease which may involve the skin, mucous membranes, hair follicles or nails. Although its pathogenesis is still unknown, there is evidence that immunological mechanisms, infections, genetic predisposition, allergic reactions to drugs, psychogenic disorders and some chronic and endocrine diseases may play important roles $[1,2]$. It has been reported that LP shows cancerous characteristics, but its neoplastic transformation rate in skin is low; while oral LP increases the risk of squamous cell carcinoma [3,4], and the World Health Organization (WHO) has classified oral LP as "potentially malignant lesions" [5]. Studies have shown that many oncogenes or anti-oncogenes, such as Bcl-2, p53 and Ki-67 were abnormally expressed in LP lesions [6-8], which indicates its potential correlations with tumors.

Yes-Associated Protein (YAP), a key component of the Hippo signaling pathway, plays an important role in organ size control, cell proliferation and apoptosis [9]. In recent years, it has been found to be up-regulated in many malignant tumors, such as liver cancer, breast cancer, lung cancer, gastric cancer, colon cancer, etc. Studies have shown that it may be through inhibiting apoptosis, regulating cell cycle, promoting the migration and invasion abilities and inhibiting stem cell differentiation that YAP is involved in the occurrence and development of many tumors, so it is considered as an oncogene [10-12]. However, to the best of our knowledge, its role in LP has not been investigated. The aim of the present study was to demonstrate the expression of YAP in LP by immunochemistry, and to infer the potential role of YAP in the pathogenesis and malignant transformation of LP.

\section{Materials and Methods}

\section{Patient samples}

LP specimens of 30 cases were obtained from the tissue bank of Department of Dermatology, the Second Affiliated Hospital of Xi'an Jiaotong University. Among them, 14 were male, and 16 were female, aging 26-79 years with average age of (54.36 \pm 15.54 ) years. Meanwhile, 30 cases of normal skin specimens were obtained from cosmetic surgery, including 18 males and 12 females, aging 43-89 years with the average age of (67.73 \pm 12.18) years. The baseline information of the two groups was not significantly different and was comparable. All the LP cases were clinically and pathologically verified. Informed consent for tissue procurement was obtained from all patients before study initiation, and Ethics approval was obtained from the Institutional Ethics Committee of Xi'an Jiaotong University.

\section{Immunohistochemistry}

We analyzed YAP expressions in LP and normal skin tissues via immunohistochemistry. The specimens were embedded in paraffin and prepared into pathological slices. Immunohistochemical staining was performed using a standard immunoperoxidase staining procedure [13], with rabbit antihuman monoclonal YAP antibody as primary antibody (\#4912, Cell Signaling Technology, Danvers, MA, USA) and biotin-labeled goat anti-rabbit IgG as secondary antibody (BA1003, Wuhan Boster Biological Technology, Wuhan, China). A negative control was established using Phosphate-Buffered Saline (PBS) instead of the primary antibody. The staining results were evaluated under microscope by two independent pathologists and quantitated based on the following scoring system: (1) percentage of positive cells: $\leq 5 \%$ scored $0,6 \% \sim 25 \%$ scored $1,26 \% \sim 50 \%$ scored 2 , $51 \% \sim 75 \%$ scored 3, > 75\%, scored 4; (2) staining intensity: 
colorless scored 0 , light yellow scored 1 , yellowish brown scored 2 and chocolate brown scored 3. The overall score for each microscopic field was calculated by the product of the two scores. The average score from five fields was taken as the final score of YAP expression for each slide. Score 0 was considered as negative $(-)$; score 1-4 were considered as weakly positive (+); score 5-8 were considered as positive $(++)$; score $9-12$ were considered as strong positive $(+++)$.

\section{Statistical analysis}

Data were analyzed using software SPSS 17.0 (SPSS, Inc., Chicago, IL, USA). Chi-square test was used. All data are presented as the Mean \pm SEM. $\mathrm{P}<0.05$ was considered as statistically significant.

\section{Results}

The results of immunohistochemistry showed both nuclear and cytoplasmic staining of YAP. Among 30 specimens of normal skin tissues, YAP was found to be weakly expressed in the basal layers of the skin (Figure 1) with a positive rate of $26.67 \%(8 / 30)$; while in LP, YAP was expressed in the lower layers of the stratum spinosum and some of the infiltrating lymphocytes (Figure 2) with a positive rate of $56.67 \%(17 / 30)$. The difference between the two groups was statistically significant $(\chi 2=5.554, P=0.018$ $<0.05)$ (Table 1).

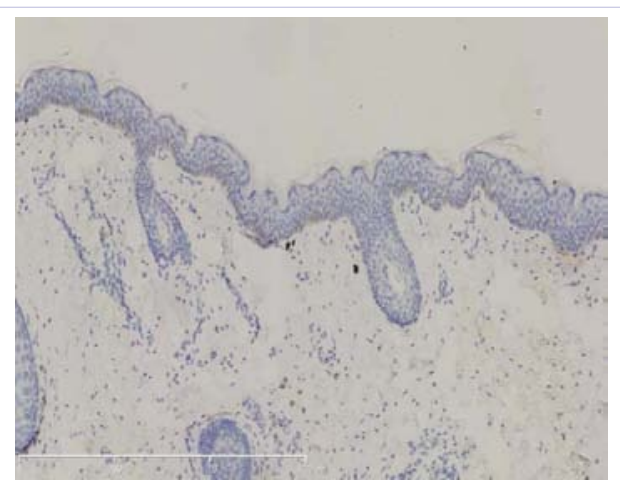

Figure 1: Expression of YAP in normal skin tissues (bar length = $600 \mu \mathrm{m})$.

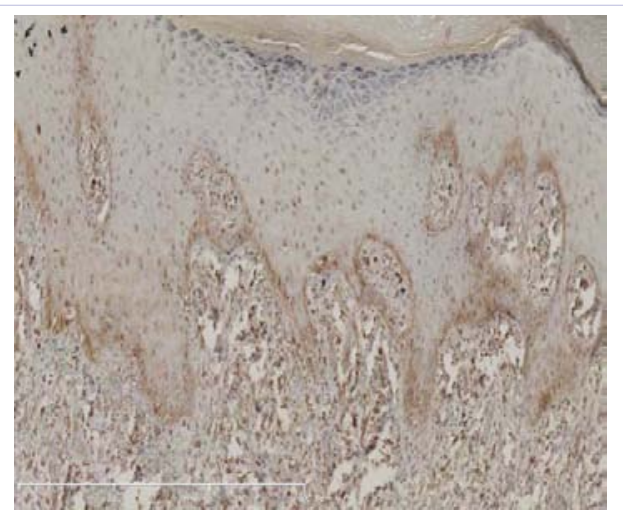

Figure 2: Expression of YAP in lichen planus tissues (bar length = $600 \mu \mathrm{m})$.

\section{Discussion}

Lichen Planus (LP) is a common chronic inflammatory disease. Skin LP has a relatively low incidence to develop cancer. The hypertrophic or ulcerative lesions may transform into cutaneous squamous cell carcinoma [14-16]; while the oral LP increases the risk of oral squamous cell carcinoma, so WHO has classified it as precancerous lesions [17]. Therefore, early detection of the precancerous lesions, so that patients receive timely treatment, has become an important issue for clinicians.

Yes-Associated Protein (YAP) was originally identified in Drosophila [18]. Its molecular weight is $65 \mathrm{kDa}$, so it is also called YAP65 [19]. In recent years, it was found to be upregulated in many tumors [10-12]. It may be through regulating cell proliferation; apoptosis, Epithelial Mesenchymal Transition (EMT), intercellular contact inhibition and self-renewal of stem cells that YAP participate in the occurrence and development of tumors [20-24], so it is considered as an oncogene. By immunohistochemical staining, researchers have found that YAP is upregulated in a wide variety of human cancers including liver cancer [25], breast cancer [26], lung cancer [27], gastric cancer, esophageal cancer [28] and colorectal cancer [29]. YAP was also found to be correlated with some skin and mucosa tumors, such as oral squamous cell carcinoma [30], malignant melanoma $[31,32]$ and head and neck cancers [33]. But so far, little is known about expression of YAP in LP. Therefore, the present study is of great significance for the expression and potential roles of YAP in LP.

In the present study, the expression of YAP was detected by immunohistochemical method. It was found to be expressed in normal skin tissues, but only confined to the basal layers of the epidermis and the positive rate is $26.67 \%$ (8/30). However, in LP lesions, it could also be expressed in the lower layers of the stratum spinosum and some of the infiltrating lymphocytes, and the positive rate increased to $56.67 \%(17 / 30)$. The results showed a significant difference between the two groups. This evidence suggests that the pathogenesis of LP is closely correlated with YAP. Because its proliferation promoting effects of YAP, some studies have suggested that it may play roles in chronic inflammation and wound healing [34,35], which are also pathological characteristics of ulcerative or erosive skin tumors as well as LP. And from the oncogenic effects of YAP in other tumors, we inferred that it may play a role in whether the neoplastic transformation from LP to squamous cell carcinoma, or simply reactivity to superficial erosion and chronic inflammation of LP. But we lack the long-term follow-up data. Such limitations prevented us from reaching definitive and clear conclusions about the influence of YAP on LP neoplastic transformation.

In conclusion, the abnormal positive expression of YAP is significantly elevated in LP lesions. Further studies are still needed to verify whether YAP could be used as an important indicator of LP canceration and thus provide evidence to support the early diagnosis and treatment of LP with malignant potential. 
Table 1: Expression of YAP in normal skin and lichen planus Case.

\begin{tabular}{|c|c|c|c|c|c|c|c|c|}
\hline \multirow{2}{*}{ Group } & \multirow{2}{*}{ Case } & \multicolumn{4}{|c|}{ Expression grade } & \multirow{2}{*}{$\begin{array}{c}\text { Positive rate } \\
\text { (\%) }\end{array}$} & \multirow{2}{*}{$\chi^{2}$} & \multirow{2}{*}{ P-value } \\
\hline & & - & + & ++ & +++ & & & \\
\hline Normal skin & 30 & 22 & 6 & 2 & 0 & 26.67 & 5.554 & 0.018 \\
\hline Lichen planus & 30 & 13 & 10 & 6 & 1 & 56.67 & & \\
\hline
\end{tabular}

\section{Acknowledgement}

This work was supported by grants from the National Natural Science Foundation of China (81573055, 81371732), and partially supported by Funds of Shaanxi Province (2015KTCL0310) and 2nd Hospital of Xi'an Jiaotong University.

\section{References}

1. Friedman P, Sabban EC, Marcucci C, Peralta R, Cabo H. Dermoscopic findings in different clinical variants of lichen planus. Is dermoscopy useful? Dermatol Pract Concept. 2015;5(4):51-55. doi: 10.5826/ dpc.0504a13.

2. Alaizari NA, Al-Maweri SA, Al-Shamiri HM, Tarakji B, Shugaa-Addin B Hepatitis c virus infections in oral lichen planus: a systematic review and meta-analysis. Aust Dent J. 2015. doi: 10.1111/adj.12382.

3. Bombeccari GP, Guzzi G, Tettamanti M, Giannì AB, Baj A, Pallotti F, Spadari F. Oral lichen planus and malignant transformation: a longitudinal cohort study. Oral Surg Oral Med Oral Pathol Oral Radiol Endod. 2011;112(3):328-334. doi: 10.1016/j.tripleo.2011.04.009.

4. Shen ZY, Liu W, Feng JQ, Zhou HW, Zhou ZT. Squamous cell carcinoma development in previously diagnosed oral lichen planus: de novo or transformation? Oral Surg Oral Med Oral Pathol Oral Radiol Endod. 2011;112(5):592-596. doi: 10.1016/j.tripleo.2011.05.029.

5. Warnakulasuriya S, Johnson NW, van der Waal I. Nomenclature and classification of potentially malignant disorders of the oral mucosa. J Oral Pathol Med. 2007;36(10):575-580.

6. Bal N, Tuncer I, Baba M, Bolat F. Bcl-2 expression in dermal lymphocytes in lichen planus and psoriasis vulgaris. J Eur Acad Dermatol Venereol. 2008;22(5):640-642. doi: 10.1111/j.1468-3083.2007.02434.x

7. Leyva-Huerta ER, Ledesma-Montes C, Rojo-Botello RE, Vega-Memije E. P53 and bcl-2 immunoexpression in patients with oral lichen planus and oral squamous cell carcinoma. Med Oral Patol Oral Cir Bucal. 2012;17(5):e745-750. doi: 10.4317/medoral.18013

8. Dummer R, Michie SA, Kell D, Gould JW, Haeffner AC, Smoller BR, Warnke RA, Wood GS. Expression of bcl-2 protein and Ki-67 nuclear proliferation antigen in benign and malignant cutaneous T-cell infiltrates. J Cutan Pathol. 1995;22(1):11-17.

9. Edgar BA. From cell structure to transcription: Hippo forges a new path. Cell. 2006;124(2):267-273

10. Lorenzetto E, Brenca M, Boeri M, Verri C, Piccinin E, Gasparini P, Facchinetti F, Rossi S, Salvatore G, Massimino M, Sozzi G, Maestro R, Modena P. YAP1 acts as oncogenic target of 11q22 amplification in multiple cancer subtypes. Oncotarget, 2014;5(9):2608-2621.

11. Steinhardt AA, Gayyed MF, Klein AP, Dong J, Maitra A, Pan D, Montgomery EA, Anders RA. Expression of Yes-associated protein in common solid tumors. Hum Pathol. 2008;39(11):1582-1589. doi: 10.1016/j.humpath.2008.04.012.

12. Chan SW, Lim CJ, Chen L, Chong YF, Huang C, Song H, Hong W. The Hippo pathway in biological control and cancer development. J Cell
Physiol, 2011;226(4):928-939. doi: 10.1002/jcp.22435.

13. Pinheiro C, Longatto-Filho A, Scapulatempo C, Ferreira L, Martins S, Pellerin L, Rodrigues M, Alves VA, Schmitt F, Baltazar F. Increased expression of monocarboxylate transporters 1, 2, and 4 in colorectal carcinomas. Virchows Arch. 2008;452(2):139-146. doi: 10.1007/ s00428-007-0558-5.

14. Ghosh S, Kotne S, Ananda Rao PB, Turlapati SP, Kumar Soren D. Squamous cell carcinoma developing in a cutaneous lichen planus lesion: a rare case. Case Rep Dermatol Med. 2014;2014:3. doi. org/10.1155/2014/205638

15. Ardabili M, Gambichler T, Rotterdam S, Altmeyer P, Hoffmann K, Stücker M. Metastatic cutaneous squamous cell carcinoma arising from a previous area of chronic hypertrophic lichen planus. Dermatol Online J. 2003;9(1):10.

16. Ali NM, Bhat R, Rao SB. Concurrent Presentation of Erythrodermic Lichen Planus and Squamous Cell Carcinoma: Coincidence or Malignant Transformation? Indian J Dermatol. 2015;60(5):457-460. doi: 10.4103/0019-5154.164355

17. Silverman S Jr. Oral lichen planus: a potentially premalignant lesion. J Oral Maxillofac Surg. 2000;58(11):1286-1288.

18. Sudol M. Yes-associated protein (YAP65) is a proline-rich phosphoprotein that binds to the SH3 domain of the Yes protooncogene product. Oncogene. 1994;9(8):2145-2152.

19.Zeng $\mathrm{Q}$, Hong $\mathrm{W}$. The emerging role of the hippo pathway in cell contact inhibition, organ size control, and cancer development in mammals. Cancer Cell. 2008;13(3):188-192. doi.org/10.1016/j.ccr.2008.02.011

20. Danovi SA, Rossi M, Gudmundsdottir K, Yuan M, Melino G, Basu S. Yes-associated protein (YAP) is a critical mediator of c-Jun-dependent apoptosis. Cell Death Differ. 2008;15(1):217-219.

21. Yuan M, Tomlinson V, Lara R, Holliday D, Chelala C, Harada T, et.al. Yes-associated protein (YAP) functions as a tumor suppressor in breast. Cell Death Differ. 2008;15(11):1752-1759. doi: 10.1038/ cdd.2008.108

22. Overholtzer M, Zhang J, Smolen GA, Muir B, Li W, Sgroi DC, et.al . Transforming properties of YAP, a candidate oncogene on the chromosome 11q22 amplicon. Proc Natl Acad Sci U S A. 2006;103(33):12405-12410. doi: 10.1073/pnas.0605579103

23. Beverdam A, Claxton C, Zhang X, James G, Harvey KF, Key B. Yap controls stem/progenitor cell proliferation in the mouse postnatal epidermis. J Invest Dermatol. 2013;133(6):1497-1505. doi: 10.1038/ jid.2012.430

24. Jansson L, Larsson J. Normal hematopoietic stem cell function in mice with enforced expression of the Hippo signaling effector YAP1. PLoS One. 2012;7(2):e32013. doi: 10.1371/journal.pone.0032013.

25.Xu MZ, Yao TJ, Lee NP, Ng IO, Chan YT, Zender L, et.al. Yes-associated protein is an independent prognostic marker in hepatocellular carcinoma. Cancer. 2009; 115(19): 4576-4585. doi: 10.1002/ cncr.24495. 
26. Vlug EJ, van de Ven RA, Vermeulen JF, Bult P, van Diest PJ, Derksen PW. Nuclear localization of the transcriptional coactivator YAP is associated with invasive lobular breast cancer. Cell Oncol (Dordr). 2013;36(5):375-384. doi: 10.1007/s13402-013-0143-7.

27. Wang Y, Dong Q, Zhang Q, Li Z, Wang E, Qiu X. Overexpression of yesassociated protein contributes to progression and poor prognosis of non-small-cell lung cancer. Cancer Sci. 2010;101(5):1279-1285. doi: 10.1111/j.1349-7006.2010.01511.x.

28. Lam-Himlin DM, Daniels JA, Gayyed MF, Dong J, Maitra A, Pan D, et.al The hippo pathway in human upper gastrointestinal dysplasia and carcinoma: a novel oncogenic pathway. Int J Gastrointest Cancer. 2006;37(4):103-109. doi: 10.1007/s12029-007-0010-8.

29. Wang L, Shi S, Guo Z, Zhang X, Han S, Yang A, et.al . Overexpression of YAP and TAZ is an independent predictor of prognosis in colorectal cancer and related to the proliferation and metastasis of colon cancer cells. PLoS One. 2013;8(6):e65539. doi: 10.1371/journal. pone.0065539.
30. Li SY, Hu JA, Wang HM. Expression of Yes--associated protein 1 gene and protein in oral Squamous cell carcinoma. Chin Med J (Engl). 2013;126(4):655-658.

31. Nallet-Staub F, Marsaud V, Li L, Gilbert C, Dodier S, Bataille V,et.al. Pro-Invasive Activity of the Hippo Pathway Effectors YAP and TAZ in Cutaneous Melanoma. J Invest Dermatol. 2014;134(1):123-132. doi: 10.1038/jid.2013.319.

32. Menzel M, Meckbach D, Weide B, Toussaint NC, Schilbach K, Noor $\mathrm{S}$, et.al. In melanoma, Hippo signaling is affected by copy number alterations and YAP1 overexpression impairs patient survival. Pigment Cell Melanoma Res. 2014;27(4):671-673. doi: 10.1111/ pcmr.12249.

33. Jerhammar F, Johansson AC, Ceder R, Welander J, Jansson A, Grafström $\mathrm{RC}$, et.al . YAP1 is a potential biomarker for cetuximab resistance in head and neck cancer. Oral Oncol. 2014;50(9):832-839. doi: 10.1016/j. oraloncology.2014.06.003. 